\title{
The Effect of the False Acetabulum on Femoral Proximal Medullary Canal in Unilateral Crowe Type IV Developmental Dislocation of the Hip
}

This article was published in the following Dove Press journal: Therapeutics and Clinical Risk Management

\author{
Yinqiao Du (1)* \\ Tiejian Li* \\ Jingyang Sun \\ Ming $\mathrm{Ni}$ \\ Yonggang Zhou \\ Department of Orthopedics, Chinese \\ People's Liberation Army General \\ Hospital, Beijing I00853, People's \\ Republic of China \\ *These authors contributed equally to \\ this work
}

Purpose: To investigate the effect of the false acetabulum on femoral proximal medullary canal in unilateral Crowe type IV developmental dislocation of the hip (DDH) patients on anteroposterior radiographs.

Patients and Methods: We measured the following parameters of DDH and contralateral normal hip $(\mathrm{CNH})$ of proximal morphology of femurs on 65 patients with unilateral Crowe type IV DDH (30 hips with no false acetabulum (type IVA) and 35 hips with a false acetabulum (type IVB)) in our hospital between September 2009 and July 2019 on anteroposterior radiographs: the widths of medullary canals at $20 \mathrm{~mm}$ above the center of lesser trochanter (CLT), $20 \mathrm{~mm}$ below the CLT and the isthmus. Canal flare index (CFI), metaphyseal canal flare index (MCFI), diaphyseal canal flare index (DCFI) were calculated.

Results: The values of CFI of DDH and CNH in unilateral type IVA patients were 2.8 and 4.4 , respectively $(p<0.001)$, and those in type IVB patients were 3.9 and 4.6 , respectively $(p<0.001)$. The MCFIs of DDH and CNH in type IVA group were 2.2 and 2.3, respectively $(p=0.032)$, and those in type IVB group were 2.4 and 2.4 , respectively $(p=0.242)$. The DCFIs of DDH and CNH in type IVA group were 1.3 and 1.9 , respectively $(p<0.001)$, and those in type IVB group were 1.7 and 1.9 , respectively $(p=0.002)$.

Conclusion: The false acetabulum stimulated the development of the proximal femur in Crowe type IV DDH. The variation of the femoral proximal medullary canal in type IVA DDH mainly occurred at the metaphyseal and proximal diaphyseal levels, and that in type IVB DDH mainly occurred at the proximal diaphyseal level.

Keywords: developmental dysplasia of the hip, Crowe type IV, femoral morphology, total hip arthroplasty

\section{Introduction}

Developmental dysplasia of the hip (DDH) describes a whole range of abnormalities involving the developing hip including subluxation or irreducible dislocation, instability, and dysplasia of femur and acetabulum. ${ }^{1-3} \mathrm{DDH}$ is a recognized cause of secondary arthritis which could result in eventual total hip arthroplasty (THA) to alleviate pain and improve function. . $^{2,5}$

Many classification systems have been proposed to characterize DDH in adults. ${ }^{6-8}$ The Crowe classification system is recommended in most published literatures, ${ }^{4,9-11}$ because it could be suggestive and instructive for surgery. In Crowe type IV DDH, two kinds of dysplasia are commonly observed: one with no false acetabulum and the other with a false acetabulum. ${ }^{12}$ In our institute, Zhou et al. classified these two kinds of
Correspondence: Yonggang Zhou; Ming $\mathrm{Ni}$ Email ygzhou301@163.com; niming30I@I63.com 
Crowe type IV DDH as type IVA and type IVB hips. ${ }^{3}$ The presence of a false acetabulum is an important factor determining shortening subtrochanteric osteotomy (SSTO) application in THA for Crowe type IV DDH. ${ }^{3,9}$ The presence and absence of a false acetabulum in Crowe type IV DDH are associated with different stress patterns and different soft tissue conditions around the hip, which lead to various morphologic abnormalities with type IV DDH.,12 An understanding of femoral proximal medullary morphologic abnormalities will aid the surgeon in preparing for the femoral implants and the complexity of the DDH. ${ }^{13}$

There have been few earlier studies of the morphology of dysplastic femur in type IV DDH. A straight, narrow medullary canal and excessive high neck-shaft angles are very common. ${ }^{12-15} \mathrm{Xu}$ et al. ${ }^{12}$ had reported the femoral proximal medullary morphologic abnormalities in the presence and absence of a false acetabulum of high DDH according to the classification system of Hartofilakidis et al. ${ }^{6,8}$ However, their study did not compare the normal control hip.

We investigated whether adults patients with unilateral Crowe type IV DDH have morphologic differences between proximal femurs in type IVA, type IVB and the contralateral normal hip $(\mathrm{CNH})$ on plain radiographs. The hypothesis was that the false acetabular would stimulate the development of proximal femur in Crowe type IV DDH.

\section{Patients and Methods}

We retrospectively reviewed the anteroposterior (AP) radiographic data of all patients with unilateral Crowe type IV DDH, who were admitted to our hospital for THA between September 2009 and July 2019. The contralateral hip of each patient was normal (the Shenton line was intact, the centeredge angle of Wiberg and acetabular index were normal or nearly normal, and there was no hip osteoarthritis). ${ }^{16}$ After excluding 31 patients for the following reasons: 11 for prior hip surgery, eight with a dysplasia attributable to infection and trauma, 12 for incomplete radiographs, a total of 65 patients with unilateral Crowe type IV DDH were investigated and divided into two groups based on the Crowe and Zhou classifications: ${ }^{3,7} 38$ type IVA patients and 30 type IVB patients (Figure 1 and Table 1). Ethical approval was obtained from the review board in our hospital.

\section{Radiographic Measurement}

All radiographs were performed in the supine position. The quality of radiographs was checked to ensure bilateral obturator foramens were the same size, the tip of each coccyx was approximately $4 \mathrm{~cm}$ above the upper level of the pubic symphysis, and the lesser trochanter could been seen. ${ }^{12}$

The following parameters were measured for each femur: The widths of medullary canals were measured at $20 \mathrm{~mm}$ above the center of the lesser trochanter (CLT), $20 \mathrm{~mm}$ below the CLT and the isthmus on the AP and lateral radiographs. ${ }^{17,18}$ The other parameters were also measured on the AP radiographs: height of the center of femoral head, which was defined as the vertical distance from the center of femoral head to the level of the interteardrop line; medial offset of femoral head, which was defined as the perpendicular distance from the center of the femoral head to the femoral medullary axis; ${ }^{15}$ and neck-shaft angle. Canal flare index (CFI) was calculated
A

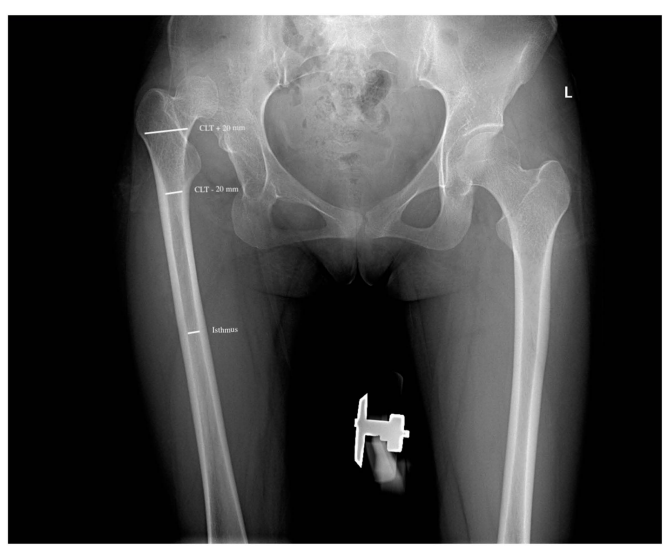

B

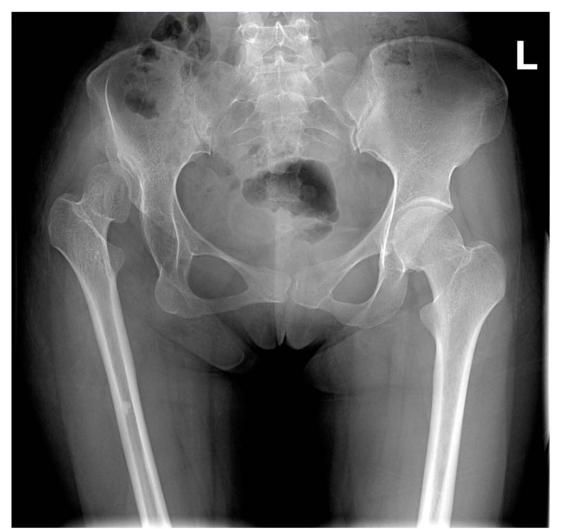

Figure I (A) A unilateral Crowe type IV DDH (right side) with no false acetabulum on AP radiograph (type IVA). (B) A unilateral Crowe type IV DDH (right side) with a false acetabulum on AP radiograph (type IVB).

Abbreviation: CLT, the center of the lesser trochanter. 
Table I Patient Demographics

\begin{tabular}{|l|l|l|l|}
\hline Characteristics & $\begin{array}{l}\text { Type IVA } \\
(\boldsymbol{n}=\mathbf{3 0})\end{array}$ & $\begin{array}{l}\text { Type IVB } \\
(\boldsymbol{n}=\mathbf{3 5})\end{array}$ & $\boldsymbol{P}$ value \\
\hline Gender (F/M) & $28 / 2$ & $34 / 1$ & 0.466 \\
Age (years) & $38.2 \pm 12.9$ & $39.1 \pm 10.26$ & 0.567 \\
Height (cm) & $159.2 \pm 6.9$ & $157.7 \pm 6.9$ & 0.682 \\
Weight (kg) & $57.4 \pm 9.7$ & $56.5 \pm 11.5$ & 0.745 \\
BMI & $22.6 \pm 2.9$ & $22.7 \pm 3.9$ & 0.969 \\
Side (R/L) & $18 / 12$ & $14 / 21$ & 0.108 \\
\hline
\end{tabular}

Abbreviations: F, female; M, male; R, right; L, light; BMI, body mass index.

as the ratio between the widths of the medullary canal at the level of the $20 \mathrm{~mm}$ above the CLT and the isthmus on the AP radiographs. ${ }^{19}$ Metaphyseal canal flare index (MCFI) was calculated as the ratio between the widths of the medullary canal at the levels of $20 \mathrm{~mm}$ above and $20 \mathrm{~mm}$ below the $\mathrm{CLT}^{20}$ Diaphyseal canal flare index (DCFI) was calculated as the ratio between the widths of the medullary canal at the level of the $20 \mathrm{~mm}$ below the CLT and the isthmus. ${ }^{21}$

Two independent investigators (YQD and JYS), who were familiar with digital measurement, performed the radiographic measurements. All of the measurements were repeated four weeks later to assess the intra-observer reliability. These intraclass correlation coefficients (ICCs) for inter-observer (0.82 to 0.91$)$ and intraobserver $(0.81$ to 0.88$)$ reliability indicated high reproducibility.

\section{Statistical Analysis}

Categorical data were compared using a chi-squared test. The paired samples $t$-test or Wilcoxon test was used to compare continuous data between the DDH and CNH. The independent-samples $t$-test or Mann-Whitney $U$-test was used to compare continuous data between the type IVA and type IVB groups. All tests were performed using SPSS version 26 (IBM Corp., Armonk, NY). A $p$-value $<0.05$ was considered significant in all analysis.

\section{Results}

The widths of medullary canals of femur at $20 \mathrm{~mm}$ above the center of the lesser trochanter (CLT), $20 \mathrm{~mm}$ below the CLT and the isthmus on the AP and lateral radiographs are see in Tables 2 and 3. The femoral proximal medullary canal of type IVA and type IVB DDH was narrower compared with that of the $\mathrm{CNH}$. The femoral medullary canal of type IVA was stovepipe-shaped and those of type IVB DDH and CNH were normal, as indicated by the values of CFI (type IVA: DDH: 2.8 and $\mathrm{CNH}$ : 4.4, $p<0.001$; type IVB: DDH: 3.9 and CNH: 4.6, $p<0.001)$. There was also a significant difference in CFI between the type IVA and IVB DDHs $(p<0.001)$. The MCFIs of DDH and CNH in type IVA group were 2.2 and 2.3, respectively $(p=0.032)$, and those in type IVB group were 2.4 and 2.4 , respectively $(p=0.242)$. There was no significant difference in MCFI between the type IVA and IVB DDHs $(p=0.065)$. The DCFIs of DDH and

Table 2 The Widths of Medullary Canals of Femur on the AP Radiographs

\begin{tabular}{|l|l|l|l|l|l|l|l|}
\hline \multirow{2}{*}{ Parameter } & \multicolumn{2}{|l|}{ Type IVA } & \multicolumn{2}{l|}{ Type IVB } & \multirow{2}{*}{ * $^{*}$} \\
\cline { 2 - 8 } & DDH & CNH & $\boldsymbol{P}$ & DDH & CNH & $\boldsymbol{P}$ \\
\hline $\mathrm{CLT}+20 \mathrm{~mm}$ & $26.3 \pm 3.0$ & $41.9 \pm 5.1$ & $<0.001$ & $36.9 \pm 5.9$ & $41.2 \pm 3.6$ & $<0.001$ & $<0.001$ \\
$\mathrm{CLT}-20 \mathrm{~mm}$ & $12.6 \pm 3.1$ & $18.2 \pm 3.5$ & $<0.001$ & $16.0 \pm 3.5$ & $17.1 \pm 2.2$ & 0.023 & $<0.001$ \\
Isthmus & $9.8 \pm 1.9$ & $9.9 \pm 1.9$ & 0.768 & $9.8 \pm 2.0$ & $9.3 \pm 1.8$ & 0.054 & 0.959 \\
\hline
\end{tabular}

Note: ${ }^{*} p$ means the differences between the cortical widths of type IVA DDH and type IVB DDH.

Abbreviations: AP, anteroposterior; $\mathrm{DDH}$, developmental dislocation of the hip; $\mathrm{CNH}$, contralateral normal hip; $\mathrm{CLT}$, the center of the lesser trochanter.

Table 3 The Widths of Medullary Canals of Femur on the Lateral Radiographs

\begin{tabular}{|c|c|c|c|c|c|c|c|}
\hline \multirow[t]{2}{*}{ Parameter } & \multicolumn{3}{|l|}{ Type IVA } & \multicolumn{3}{|l|}{ Type IVB } & \multirow[t]{2}{*}{$\mathbf{p}^{*}$} \\
\hline & DDH & CNH & $p$ & DDH & CNH & $p$ & \\
\hline $\mathrm{CLT}+20 \mathrm{~mm}$ & $29.01 \pm 3.85$ & $33.26 \pm 4.64$ & $<0.001$ & $33.68 \pm 4.09$ & $34.63 \pm 4.24$ & $0.25 I$ & $<0.001$ \\
\hline CLT - $20 \mathrm{~mm}$ & $15.13 \pm 2.93$ & $|8.7| \pm 3.27$ & $<0.001$ & $18.52 \pm 3.72$ & $19.28 \pm 2.84$ & 0.139 & $<0.001$ \\
\hline Isthmus & $13.30 \pm 3.29$ & $11.70 \pm 2.99$ & $<0.001$ & $12.85 \pm 2.60$ & $12.3 \mid \pm 2.74$ & 0.235 & 0.542 \\
\hline
\end{tabular}

Notes: Values are expressed as mean \pm SD. *p means the differences between the medullary canal widths of type IVA DDH and type IVB DDH. Abbreviations: DDH, developmental dislocation of the hip; $\mathrm{CNH}$, contralateral normal hip; CLT, the center of the lesser trochanter. 
Table 4 Parameters of Femur on the AP Radiographs

\begin{tabular}{|c|c|c|c|c|c|c|c|}
\hline \multirow[t]{2}{*}{ Parameter } & \multicolumn{3}{|l|}{ Type IVA } & \multicolumn{3}{|l|}{ Type IVB } & \multirow[t]{2}{*}{$\mathbf{p}^{*}$} \\
\hline & DDH & CNH & $p$ & DDH & CNH & p & \\
\hline CFI & $2.8 \pm 0.6$ & $4.4 \pm 1.0$ & $<0.001$ & $3.9 \pm 0.9$ & $4.6 \pm 1.0$ & $<0.001$ & $<0.001$ \\
\hline MCFI & $2.2 \pm 0.6$ & $2.3 \pm 0.4$ & 0.032 & $2.4 \pm 0.4$ & $2.4 \pm 0.3$ & 0.242 & 0.065 \\
\hline DCFI & $1.3 \pm 0.2$ & $1.9 \pm 0.3$ & $<0.001$ & $1.7 \pm 0.3$ & $1.9 \pm 0.3$ & 0.002 & $<0.001$ \\
\hline Height of the center of the femoral head $(\mathrm{mm})$ & $75.3 \pm 11.7$ & $18.1 \pm 3.5$ & $<0.001$ & $59.9 \pm 10.7$ & $17.7 \pm 3.3$ & $<0.001$ & $<0.001$ \\
\hline Medial offset of the femoral head $(\mathrm{mm})$ & $28.3 \pm 9.1$ & $39.9 \pm 7.8$ & $<0.001$ & $30.9 \pm 6.5$ & $39.2 \pm 7.4$ & $<0.001$ & 0.190 \\
\hline Neck-shaft angle (degrees) & $127.2 \pm 11.2$ & $128.4 \pm 5.2$ & 0.598 & $132.6 \pm 7.4$ & $128.6 \pm 6.0$ & 0.017 & 0.023 \\
\hline
\end{tabular}

Notes: * $p$ means the differences between the cortical widths of type IVA DDH and type IVB DDH.

Abbreviations: AP, anteroposterior; DDH, developmental dislocation of the hip; $\mathrm{CNH}$, contralateral normal hip; CFI, canal flare index; $\mathrm{MCFI}$, metaphyseal canal flare index; DCFI, diaphyseal canal flare index.

$\mathrm{CNH}$ in type IVA group were 1.3 and 1.9 , respectively $(p<0.001)$, and those in type IVB group were 1.7 and 1.9 , respectively $(p=0.002)$. There was also a significant difference in DCFI between the type IVA and IVB DDHs $(p<0.001)$ (Table 4).

The height of the center of the femoral head was significantly higher in type IVA DDH $(75.3 \mathrm{~mm})$ compared with the IVB DDH $(59.9 \mathrm{~mm})(p<0.001)$. The medial offset of the femoral head of DDH and CNH in type IVA group were $28.3 \mathrm{~mm}$ and $39.9 \mathrm{~mm}$, respectively $(p<$ 0.001 ), and those in type IVB group were $30.9 \mathrm{~mm}$ and $39.2 \mathrm{~mm}$, respectively $(p<0.001)$. There was no significant difference in the medial offset of femoral head between the type IVA and IVB DDHs $(p=0.190)$. The neck-shaft angle of DDH and CNH in type IVA group were 127.2 and 128.4, respectively $(p=0.598)$, and those in type IVB group were 132.6 and 128.6 , respectively ( $p=0.017$ ). There was also a significant difference in neck-shaft angle between the type IVA and IVB DDHs $(p=0.023)$.

\section{Discussion}

The study hypothesis was confirmed: The false acetabulum stimulated the development of the proximal femur in Crowe type IV DDH. Although THA is often the only definitive way of restoring the normal biomechanics of the Crowe type IV DDH, these procedures are often challenging, primarily because of the associated bone deformities of the femur and acetabulum. Typically, the dysplastic femur has also been shown to have a straighter, stovepipeshaped canal and the neck-shaft angle is more valgus than that of the normal femur. ${ }^{15}$ Because of the difficulties of inserting conventional femoral prostheses into canals of distorted shape and version, THA are associated with an increased prevalence of intraoperative complications, most notably cortical fracture. ${ }^{22}$
Secondary osteoarthritis of Crowe type IV DDH are very common, resulting in the presence of a false acetabulum, which is classified as type IVB DDH. The absence of a false acetabulum without osteoarthritis is classified as type IVA DDH. ${ }^{3,9,12}$ In Crowe type IVB DDH, the load of the pelvis is transmitted to the femur through the false acetabulum, which stimulates the development of the proximal femur. However, Crowe type IVA DDH may carry similar weight during walking with soft tissue connections, without direct bone to bone contact. Morphological studies in patients with the two subtypes of Crowe type IV DDH who are due to undergo THA are very important to reduce complications.

We found in our study that there was a significant difference of CFI and DCFI between DDH and CNH in the two subtypes. In type IVA patients, there was a significant difference of MCFI between DDH and $\mathrm{CNH}$. However, there was no significant difference of MCFI in type IVB patients. This revealed two important facts. The first is that the stovepipe-shaped femoral medullary canal is the most common in type IVA DDH. The second is that the variation of the femoral proximal medullary canal in type IVA DDH mainly occurred at the metaphyseal and proximal diaphyseal levels, and that in type IVB DDH mainly occurred in proximal diaphyseal level. Thus, the CFI, MCFI and DCFI in type IVA DDH were significantly smaller than those of type IVB DDH and $\mathrm{CNH}$, which indicated that the shape of the femoral proximal medullary canal in type IVA DDH was similar to that of a chimney. The shape of the femoral proximal medullary canal in type IVB DDH was similar to that of $\mathrm{CNH}$. Xu et al. ${ }^{12}$ compared the femurs in type $\mathrm{C} 1$ and type C2 DDH according to the classification system of Hartofilakidis et al. ${ }^{6,8}$ and found that for the distal part of proximal femur, the canal width was the same in types $\mathrm{C} 1$ 
and $\mathrm{C} 2$ hips, whereas in the metaphyseal region, the canals were much narrower in type $\mathrm{C} 2$ hips than type $\mathrm{C} 1$ hips. Our study also revealed the same results. Liu et al. ${ }^{21}$ revealed the narrowing is the most severe in Crowe IV group and the narrowing mainly occurred at the metaphyseal and proximal diaphyseal levels (the segment around the lesser trochanter).

We found the height of the center of the femoral head was more superior in type IVA DDH than that in type IVB DDH. The medial offset of the femoral head was shorter in type IVA DDH and type IVB DDH than in the CNH. Noble et al. ${ }^{22}$ reported the same results and revealed that the offset of Crowe IV DDH patients was significantly smaller than those from the normal hips. Liu et al. ${ }^{21}$ regarded the offset as an importantly extramedullary parameter of the proximal femur. Deficiency of the offset would weaken the power of the hip abductor and lead to poorer gait in Crowe IVA DDH patients. The neck-shaft angle is an influence factor of the offset and a very important extramedullary parameter of the proximal femur. It is commonly believed that the neck-shaft angle of DDH was larger than those of $\mathrm{CNH}^{21,23}$ On the contrary, our study revealed that this parameter did not differ significantly between the DDH and $\mathrm{CNH}$ in the Crowe IVA patients; moreover, it was interesting to note that the neck-shaft angle of the Crowe IVB DDH was significantly larger than that of the $\mathrm{CNH}$. Through analysis of the values of the medial offset of femoral head and the neck-shaft angle, we also found that the neck length of the DDH may be shorter as compared to that of the CNH. Liu et al. ${ }^{21}$ and Sugano et al. ${ }^{15}$ also reported the value of the neck length of the Crowe IV DDH was the shortest than those of the normal hip and Crowe I-III DDH. The rotational deformity of the dysplastic proximal femur is an important factor resulting in the increase of the anteversion. Noble et al. found that the primary deformity of the dysplastic femur is rotation, with an increase in anteversion of 5 to 16 degrees, depending on the degree of subluxation of the hip. ${ }^{22}$ They also thought the rotation arises within the diaphysis between the lesser trochanter and the isthmus and is not attributable to a deformity of the metaphysis. ${ }^{22}$

An understanding of these intra- and extramedullary challenges would help the surgeon in determining the optimal femoral stem choice in DDH patients. Standard cementless stem designs were suitable to treat patients with secondary osteoarthritis due to mild $\mathrm{DDH} .{ }^{23}$ Noble et al. recommended the use of modular or specially designed components to accommodate the shape of the dysplastic canal. ${ }^{22}$ In order to address these challenges, a primary modular stem, such as S-ROM femoral components (DePuy, Warsaw, IN, USA), may aid in correction and restoration of appropriate hip mechanics. ${ }^{13}$ The modular stems can essentially decouple the metaphyseal or diaphyseal fit from the version of the stem. ${ }^{13}$ In our institution, an S-ROM sleeve was widely used to match the shape of the metaphyseal medullary canal and adjust the excessive anteversion. Then an S-ROM stem was implanted to match the straight and narrow diaphyseal canal. Although the femoral proximal medullary canal of type IVB DDH was closer to the normal, the S-ROM femoral component was still recommended because of the excessive anteversion and anteroposterior diameter of the femoral proximal medullary canal. Wang et al. ${ }^{4}$ reported the satisfactory mid-term results of THA with S-ROM femoral components in Crowe IV DDH in our institution.

In our institution, the capsule was removed to expose the true acetabulum during surgery. In order to use a ceramic-on-ceramic bearing, the shell (size range 44-46 mm) was implanted at the level of the true acetabulum by reaming the acetabulum posteriorly and inferiorly. ${ }^{24}$ Gluteus minimus and gluteal sling release and iliopsoas tenotomy was performed. If hip reduction with a femoral trial stem was impossible, an SSTO would be performed for femoral shortening. The position of osteotomy was located at the distal end of sleeve. ${ }^{25}$ The osteotomy length equaled the distance between the true acetabular center and femoral head center during the trial reduction minus $15 \mathrm{~mm}$. In this study, an SSTO was expected to be performed (29 hips in 30 hips, 96.7\%) in type IVA DDH. And in type IVB DDH, an SSTO was not expected to be performed (29 hips in 35 hips, 82.9\%). A false acetabulum may be an important factor determining SSTO preformation in THA for Crowe type IVA DDH.

Some limitations of this study have already been discussed. First, all measures were based on plain radiographs not the $\mathrm{CT}$ and three-dimensional computer reconstruction models. Second, the authors studied the differences between the Crowe type IV DDH and the $\mathrm{CNH}$. However, we do not know the difference between the $\mathrm{CNH}$ of unilateral Crowe type IV DDH patients and that of the healthy person without DDH. The CFI of the healthy person was described in many studies, and CFI in the $\mathrm{CNH}$ of unilateral Crowe type IV DDH could be compared with that of normal people of the healthy person without DDH to reduce the limitations. 


\section{Conclusions}

The false acetabulum stimulated the development of the proximal femur in Crowe type IV DDH. The variation of the femoral proximal medullary canal in type IVA DDH mainly occurred at the metaphyseal and proximal diaphyseal levels, and that in type IVB DDH mainly occurred at the proximal diaphyseal level. An understanding of these morphologic challenges would help the surgeon in determining the optimal femoral stem and SSTO choice in DDH patients.

\section{Ethics Approval and Informed Consent}

The Ethics Committee of our hospital, General Hospital of Chinese People's Liberation Army, approved the study protocol. All the study participants provided written informed consent for the study.

\section{Consent for Publication}

Written informed consent was obtained from all patients for publication of this study and any accompanying images.

\section{Author Contributions}

All authors made substantial contributions to conception and design, acquisition of data, or analysis and interpretation of data; took part in drafting the article or revising it critically for important intellectual content; gave final approval of the version to be published; and agree to be accountable for all aspects of the work.

\section{Disclosure}

The authors declare that they have no conflicts of interest.

\section{References}

1. Kotlarsky P, Haber R, Bialik V, Eidelman M. Developmental dysplasia of the hip: what has changed in the last 20 years? World J Orthop. 2015;6(11):886-901. doi:10.5312/wjo.v6.i11.886

2. Seo LJ, Gabor J, Novikov D, Feng JE, Schwarzkopf R, Vigdorchik JM. Outcomes in 385 developmental dysplastic hips requiring total hip arthroplasty. Arch Orthop Trauma Surg. 2019;139 (5):723-728. doi:10.1007/s00402-019-03143-5

3. Ma HY, Zhou YG, Zheng C, et al. New classification of crowe type IV developmental dysplasia of the hip. Zhongguo Gu Shang. 2016;29 (2):119-124.

4. Wang S, Zhou Y, Ma H, Du Y, Piao S, Wu W. Mid-term results of total hip replacement with subtrochanteric osteotomy, modular stem, and ceramic surface in crowe IV hip dysplasia. Arthroplast Today. 2018;4 (3):363-369. doi:10.1016/j.artd.2017.07.003

5. Hitz OF, Flecher X, Parratte S, Ollivier M, Argenson JN. Minimum 10 -year outcome of one-stage total hip arthroplasty without subtrochanteric osteotomy using a cementless custom stem for crowe III and IV hip dislocation. J Arthroplasty. 2018;33(7):2197-2202. doi:10.10 16/j.arth.2018.02.055
6. Hartofilakidis G, Yiannakopoulos CK, Babis GC. The morphologic variations of low and high hip dislocation. Clin Orthop Relat Res. 2008;466(4):820-824. doi:10.1007/s11999-008-0131-9

7. Crowe JF, Mani VJ, Ranawat CS. Total hip replacement in congenital dislocation and dysplasia of the hip. J Bone Joint Surg Am. 1979;61 (1):15-23. doi:10.2106/00004623-197961010-00004

8. Hartofilakidis G, Stamos K, Ioannidis TT. Low friction arthroplasty for old untreated congenital dislocation of the hip. J Bone Joint Surg Br. 1988;70(2):182-186. doi:10.1302/0301-620X.70B2.3346284

9. Peng H, Zhang G, Xu C, Wang T, Wang Y. Is pseudoacetabulum an important factor determining SSTO application in total hip arthroplasty for crowe IV hips? A retrospective cohort study. J Orthop Surg Res. 2019;14(1):201. doi:10.1186/s13018-019-1216-8

10. Montalti M, Castagnini F, Giardina F, Tassinari E, Biondi F, Toni A. Cementless Total hip arthroplasty in crowe III and IV dysplasia: high hip center and modular necks. J Arthroplasty. 2018;33(6):1813-1819. doi:10.1016/j.arth.2018.01.041

11. Li Y, Zhang X, Wang Q, et al. Equalisation of leg lengths in total hip arthroplasty for patients with crowe type-IV developmental dysplasia of the hip: classification and management. Bone Joint J. 2017;99-b (7):872-879. doi:10.1302/0301-620X.99B7.BJJ-2016-1328.R1

12. Xu H, Zhou Y, Liu Q, Tang Q, Yin J. Femoral morphologic differences in subtypes of high developmental dislocation of the hip. Clin Orthop Relat Res. 2010;468(12):3371-3376. doi:10.1007/s11999010-1386-5

13. Greber EM, Pelt CE, Gililland JM, Anderson MB, Erickson JA, Peters CL. Challenges in total hip arthroplasty in the setting of developmental dysplasia of the hip. J Arthroplasty. 2017;32(9s): S38-S44. doi:10.1016/j.arth.2017.02.024

14. Tamura K, Takao M, Hamada H, Ando W, Sakai T, Sugano N. Femoral morphology asymmetry in hip dysplasia makes radiological leg length measurement inaccurate. Bone Joint J. 2019;101-b (3):297-302. doi:10.1302/0301-620X.101B3.BJJ-2018-0965.R1

15. Sugano N, Noble PC, Kamaric E, Salama JK, Ochi T, Tullos HS. The morphology of the femur in developmental dysplasia of the hip. J Bone Joint Surg Br. 1998;80(4):711-719. doi:10.1302/0301620X.80B4.0800711

16. Zhang Z, Luo D, Cheng H, Xiao K, Zhang H. Unexpected long lower limb in patients with unilateral hip dislocation. J Bone Joint Surg Am. 2018;100(5):388-395. doi:10.2106/JBJS.17.00187

17. Argenson JN, Ryembault E, Flecher X, Brassart N, Parratte S, Aubaniac JM. Three-dimensional anatomy of the hip in osteoarthritis after developmental dysplasia. J Bone Joint Surg Br. 2005;87 (9):1192-1196. doi:10.1302/0301-620X.87B9.15928

18. Robertson DD, Essinger JR, Imura S, et al. Femoral deformity in adults with developmental hip dysplasia. Clin Orthop Relat Res. 1996;327:196-206. doi:10.1097/00003086-199606000-00025

19. Noble PC, Alexander JW, Lindahl LJ, Yew DT, Granberry WM, Tullos HS. The anatomic basis of femoral component design. Clin Orthop Relat Res. 1988;235:148-165.

20. Laine H-J, Lehto MU, Moilanen T. Diversity of proximal femoral medullary canal. J Arthroplasty. 2000;15(1):86-92. doi:10.1016/ S0883-5403(00)91311-1

21. Liu S, Zuo J, Li Z, et al. Study of three-dimensional morphology of the proximal femur in developmental adult dysplasia of the hip suggests that the on-shelf modular prosthesis may not be an ideal choice for patients with crowe type IV hips. Int Orthop. 2017;41 (4):707-713. doi:10.1007/s00264-016-3248-6

22. Noble PC, Kamaric E, Sugano N, et al. Three-dimensional shape of the dysplastic femur: implications for THR. Clin Orthop Relat Res. 2003;417:27-40.

23. Innmann MM, Hasberg S, Waldstein W, et al. Are there clinically relevant anatomical differences of the proximal femur in patients with mild dysplastic and primary hip osteoarthritis? A CT-based matched-pairs cohort study. Clin Radiol. 2019;74(11):896.e817-896. e822. doi:10.1016/j.crad.2019.06.032 
24. Zhou Y, Sun C, Wang Y. New method addressing the problem of using ceramic-on-ceramic bearing in too small acetabulum of high-riding DDH patients with THA. Semin Arthroplasty. 2012;23 (4):226-231. doi:10.1053/j.sart.2012.12.006
25. Du YQ, Sun JY, Ma HY, Wang S, Ni M, Zhou YG. Leg length balance in total hip arthroplasty for patients with unilateral crowe type IV developmental dysplasia of the hip. Orthop Surg. 2020. doi:10.1111/os.12667

\section{Publish your work in this journal}

Therapeutics and Clinical Risk Management is an international, peerreviewed journal of clinical therapeutics and risk management, focusing on concise rapid reporting of clinical studies in all therapeutic areas outcomes, safety, and programs for the effective, safe, and sustained use of medicines. This journal is indexed on PubMed Central, CAS,
EMBase, Scopus and the Elsevier Bibliographic databases. The manuscript management system is completely online and includes a very quick and fair peer-review system, which is all easy to use. Visit http://www.dovepress.com/testimonials.php to read real quotes from published authors.

Submit your manuscript here: https://www.dovepress.com/therapeutics-and-clinical-risk-management-journal 